\title{
ELASTIC WAVE PROPAGATION DEVELOPMENT FOR STRUCTURAL HEALTH MONITORING
}

\author{
Wiesław M. Ostachowicz \\ *Polish Academy of Sciences, IMP PAN, Fiszera 14, PL 80-952 Gdansk, Poland \\ *Gdynia Maritime University, Faculty of Navigation, Zjednoczenia 3, PL 81-345 Gdynia, Poland
}

$\underline{\text { Summary }}$ This paper is a personal perspective of structural health monitoring technology and its applications as see from a current literature and projects. The proposed approach deals with the spectral finite element analysis method as a means of solving the wave propagation problems in structures. The change of the wave propagation process due to a damage appearance is examined by comparing the differences between the responses from damaged and undamaged structures. The influence of the damage growth for the wave propagation is also analysed. The differences in the propagating waves allow indicating the damage location and size in a very precise way.

\section{INTRODUCTION}

Undetected damage in buildings, bridges, aerospace structures, containers and other large structures will develop if undetected and in some cases result in the collapse of the whole structure. This may have costly disastrous consequences for people and the environment including deaths, injuries, fires, contamination, economic losses, legal costs etc. It has been found that in a lot of cases a small-undetected damage (crack, plastification, delamination) has been the cause for the collapse.

Damage in structures should be detected at an early stage, before it can cause any problems and so that proper decisions for repair and/or part replacement can be taken.

This paper is focused on structural health monitoring technology and its applications in aerospace and civil engineering. Better understanding of phenomena associated with propagation of elastic waves in structural elements is very important from both the theoretical and the practical point of view. Changes in propagation of elastic waves when observed in structural elements can be used for the purpose of damage detection or identification of material parameters within those elements. In recent years significant progress has been evident in this particular field of applications.

Recently, laminated composites with built-in piezoelectrics have attracted significant attention among researchers because of their potential application to controlling vibrations, suppressing noise, as well as monitoring the health of the structures. The investigated damage detection system is based on the known fact that material discontinuities affect the propagation of elastic waves in solids. The change in material characteristics, such as a local change in stiffness or inertia caused by a crack or material damage, will affect the propagation of elastic waves and will modify the received signals. Wave frequencies that are most sensitive to damage depend on the type of structure, the type of material, and the type of damage. Elastic waves are generated and sensed by an array of transducers either embedded in, or bonded to, the surface of the structure. Wave frequencies associated with the highest detection sensitivity depend, among others, on the type of the structure, the type of material, and the type of the damage.

\section{Structural Health Monitoring Technology}

SHM (Structural Health Monitoring) technology works with a built-in sensor network on a structure. These sensors provide information regarding the condition and damage state of the structures as they age. The investigated damage detection system is based on the known fact that material discontinuities affect the propagation of elastic waves in solids. The change in material characteristics, such as a local change in stiffness or inertia caused by a crack or material damage, will affect the propagation of elastic waves and will modify the received signals.

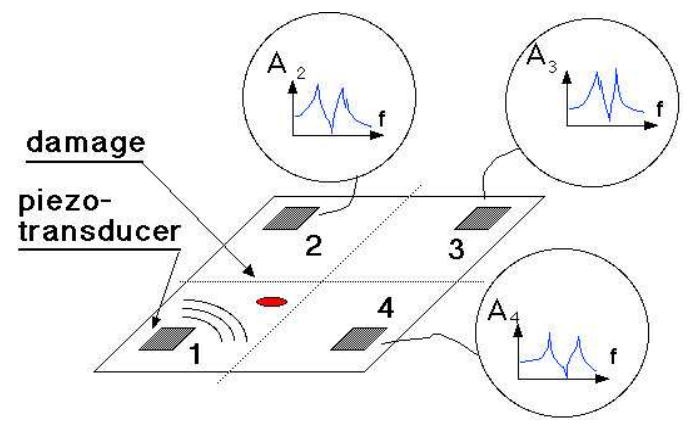

Figure 1 Built in sensor network on a plate

Wave frequencies that are most sensitive to damage depend on the type of structure, the type of material, and the type of damage. Elastic waves are generated and sensed by an array of transducers either embedded in, or bonded to, the surface 
of the structure. Wave frequencies associated with the highest detection sensitivity depend, among others, on the type of the structure, the type of material, and the type of the damage. Figure 1 presents a general concept of this technology.

\section{Spectral Finite Element Technique}

Among many frequency domain methods, the spectral method is found to be suitable due to its adaptation of displacement based FE technique and therefore enabling one to handle arbitrary skeletal structures. Spectral Element (SE) analysis has been established as a powerful method used for wave propagation. The Spectral Element Method (SEM), high-accuracy numerical method, combines the accuracy of conventional spectral methods and the geometric flexibility of finite element methods. It is the method of Fourier synthesis (or spectral analysis), where the behaviour of the signal is viewed as a superposition of many infinitely long wave trains of different periods (or frequencies). The actual response is synthesised by a judicious combination of these wave trains. Thus the problem of characterising a signal is transformed into one of determining the set of combination coefficients. These coefficients are called the Fourier transform of the signal. The problem being tackled invariably simplifies when it is expressed in terms of the Fourier transform. The last step in the analysis involves performing an inverse transform (reconstructing the signal).

This paper presents a method of wave propagation, which can be further used to detect small delaminations in beam-like and plate structures. The structures are modelled by spectral finite elements.

The approach is similar in style to that of the finite element method but with the very significant difference that the element stiffness matrix is established in the frequency domain. As a consequence, these spectrally formulated elements describe exactly the wave propagation dynamics, and in contrast to the conventional element this means that elements can span all the way from one point to another without losing fidelity.

In a simple form, the solution to a wave propagation problem can be represented as by Doyle [1]:

$$
\boldsymbol{w}=\sum_{\boldsymbol{n}} \hat{\boldsymbol{P}}_{\boldsymbol{n}}\left\{\hat{\boldsymbol{G}}_{1}\left(\boldsymbol{k}_{1 \boldsymbol{n}} \boldsymbol{x}\right)+\hat{\boldsymbol{G}}_{2}\left(\boldsymbol{k}_{2 \boldsymbol{n}} \boldsymbol{x}\right)+\ldots . . \exp \left(\boldsymbol{i} \omega_{\boldsymbol{n}} \boldsymbol{t}\right)=\sum_{\boldsymbol{n}} \hat{\boldsymbol{P}}_{\boldsymbol{n}} \hat{\boldsymbol{G}}_{1}\left(\boldsymbol{k}_{\boldsymbol{m} \boldsymbol{n}} \boldsymbol{x}\right) \exp \left(\boldsymbol{i} \omega_{\boldsymbol{n}} \boldsymbol{t}\right)\right.
$$

where: $\hat{\boldsymbol{G}}$ is the analytically derived transfer function of position $\boldsymbol{x}$. $\hat{\boldsymbol{G}}$ has different numerical values at each frequency. $\hat{\boldsymbol{P}}_{\boldsymbol{n}}$ is the amplitude spectrum and is known from input conditions or from measurements. $\widehat{\boldsymbol{P}}_{\boldsymbol{n}} \widehat{\boldsymbol{G}}_{\text {is recognised as the }}$ Fourier transform of the solution.

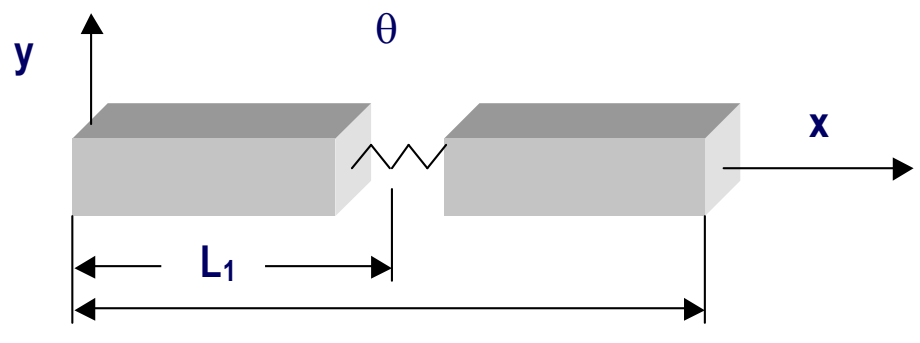

Figure 2 Rod spectral finite element

A simple spectral rod finite element with a transverse open and non-propagating crack is presented in Figure 2. The length of the element is $L$, and its area of cross-section is $A$. The crack is substituted by a dimensionless spring, which flexibility $\theta$ is calculated by using Castigliano's theorem and laws of the fracture mechanics, Krawczuk and Ostachowicz [2].

Nodal spectral displacements are assumed in the following form for the left and right part of the rod:

$$
\begin{aligned}
& \hat{u}_{1}(x)=A_{1} e^{-i k_{n} x}+B_{1} e^{-i k_{n}\left(L_{1}-x\right)} \quad \text { for } x \in\left(0, L_{1}\right) \\
& \hat{u}_{2}(x)=A_{2} e^{-i k_{n}\left(x+L_{1}\right)}+B_{2} e^{-i k_{n}\left[L-\left(L_{1}+x\right)\right]} \text { for } x \in\left(0, L-L_{1}\right)
\end{aligned}
$$

where: $\boldsymbol{L}_{\boldsymbol{1}}$ denotes the location of the crack, $\boldsymbol{L}$ is the total length of the rod and $\boldsymbol{k}_{\boldsymbol{n}}$ is the wave number calculated as follows:

$$
\boldsymbol{k}_{n}=\omega_{n} \sqrt{\frac{\rho}{E}}
$$

where: $\boldsymbol{\rho}$ is the density of the rod material, $\boldsymbol{E}$ denotes Young's modulus and $\boldsymbol{\omega}_{n}$ is a natural frequency.

The coefficients $\boldsymbol{A}_{1}, \boldsymbol{A}_{2}, \boldsymbol{B}_{1}$ and $\boldsymbol{B}_{2}$ can be calculated as a function of the nodal spectral displacements using the element boundary conditions (see Krawczuk and Ostachowicz [2]). Using boundary conditions can relate the coefficients $A_{1}$, $A_{2}$, $\boldsymbol{B}_{1}$ and $\boldsymbol{B}_{2}$ to the nodal spectral displacements. The nodal spectral forces can be determined by differentiating the spectral displacements with respect to $\boldsymbol{x}$. Finally, using the relation between nodal forces and nodal displacements the dynamic stiffness matrix of a cracked rod spectral finite element can be evaluated.

This paper presents also other applications of wave propagation, which can be further used to detect small delaminations in beam-like Krawczuk and Ostachowicz [2], Ostachowicz et al. [4], and plate structures Krawczuk et al. [3]. All considered structures are modelled by spectral finite elements. 
The SEM approach is based on global approximations of functions, i.e. the analysed functions are at first approximated using basis functions and are then exactly differentiated. As a consequence, relatively small number of elements can be used for modelling without losing accuracy. This is particularly useful for wave propagation modelling.

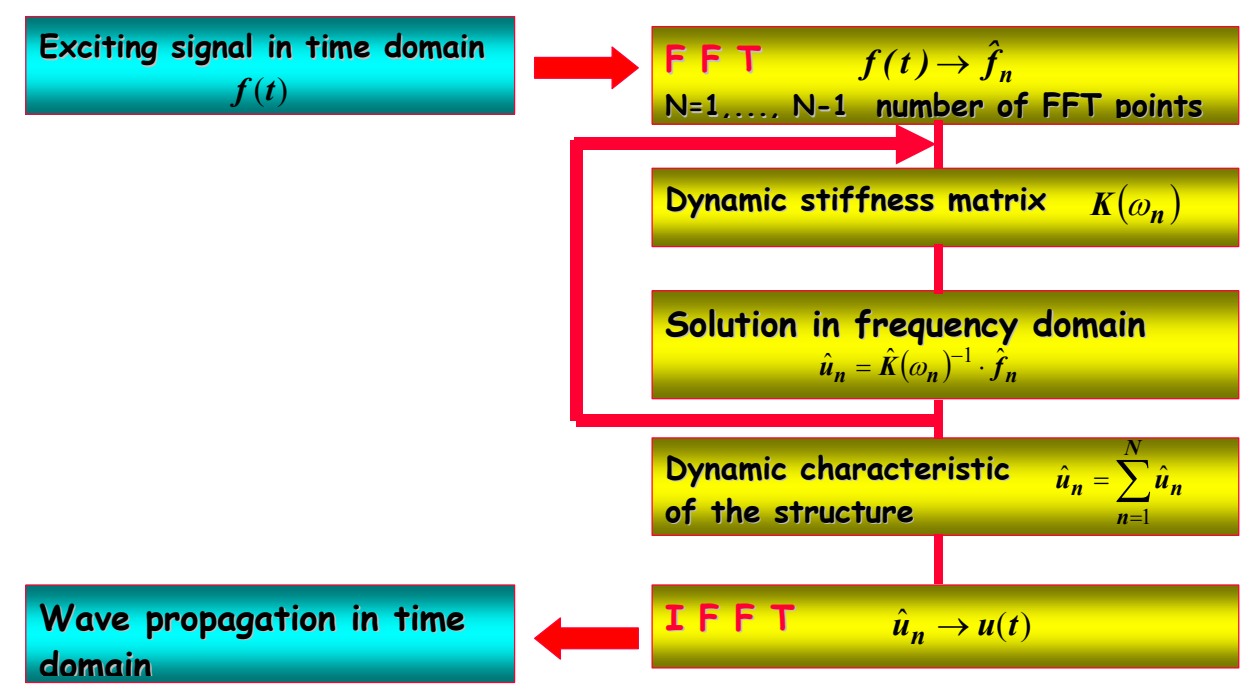

Figure 3 Flow diagram for the wave reconstruction program

The basic steps in the development of SEM can be described as follows. The governing wave equations are first transformed from the time domain to the frequency domain using a discrete Fourier transform (DFT). For computational implementation we use the FFT algorithm built into the FE code. The spatial variation is semi-explicitly obtained by solving the characteristic equation in k-space. This results in a complex shape function matrix representing the linear superposition of all the wave modes. Also, the complex dynamic strain-displacement matrix and the dynamically consistent force vector can both be derived. Following the conventional FE method, the complex dynamic stiffness matrix is then formed, and this is exact. This makes the proposed SEM an efficient model suitable for use within the framework of an automated FE method rather than a trade-off for broadband wave propagation analysis. But the fundamental difference from the conventional FE method is that all the spectral amplitudes that correspond to elemental nodal variables are evaluated at each frequency step (FFT sampling points) instead of pseudo-static variables evaluated at each time step or at each eigenfrequency. The global system is solved for unit spectral amplitude of the applied load history at each FFT sampling frequency. Therefore, computation in this way of the frequency response function (FRF) of the global system is straightforward. The time domain response is obtained using the inverse FFT.

Certainly the solution is different at each position, but once it is evaluated at a particular position then its inverse immediately gives the time history of the solution at that point. Figure 3 shows a flow diagram for the basic algorithm to propagate a wave.

\section{CONCLUSIONS}

This paper is a personal perspective of structural health monitoring technology and its applications as see from a current literature and projects. The proposed approach deals with the spectral finite element analysis method as a means of solving the wave propagation problems in structures. The change of the wave propagation process due to a damage appearance is examined by comparing the differences between the responses from damaged and undamaged structures. The influence of the damage growth for the wave propagation is also analysed. The differences in the propagating waves allow indicating the damage location and size in a very precise way.

The proposed model can easily be used for detection of damage in complicated situations, i.e. multiple delaminations located in different places.

The paper is not intended to be a comprehensive survey but merely to present a flavour of recent activity in this important subject.

\section{References}

[1] Doyle, J., Wave Propagation in Structures, Springer-Verlag, New York, 1997.

[2] Krawczuk M., and Ostachowicz W., Key Engineering Materials, Vols. 204-205, 2001, pp. 241-250.

[3] Krawczuk, M., Palacz M., and Ostachowicz W., Journal of Sound and Vibration, Vol. 264, 2003, pp. 1139-1153.

[4] Ostachowicz, W., Krawczuk, M., Cartmell, M., and Gilchrist M., Computers and Structures, Vol. 82, 2004, pp. 475-483. 